\title{
An unusual case of possible cyclic Cushing's disease in a young man with Klinefelter Syndrome
}

\author{
Thea Christoffersen¹, MBBS, Anders Juul 2 , MD, DMSc, Aase Krogh Rasmussen¹, MD, \\ DMSc, Ulla Feldt-Rasmussen ${ }^{1}$, MD, DMSc
}

1Department of Endocrinology, Rigshospitalet, Copenhagen University Hospital, Denmark ${ }^{2}$ Department of Growth and Reproduction, Rigshospitalet, Copenhagen University Hospital, Denmark

\section{Introduction}

Cushing's syndrome (CS) exist in a complicated variant characterised by alternating periods of normo - and hypercortisolism, Cyclic Cushing's syndrome. The so-called cycles of hypercortisolism can occur regularly or irregularly with intercyclic phases ranging from days to years. The fluctuating clinical picture and discrepant biochemical findings make cyclic CS extremely hard to diagnose. The pathophysiology of cyclic CS remain largely unknown.

Klinefelter syndrome is a chromosomal condition that affects male physical and cognitive development. Typically, affected individuals have smaller testes and lower testosterone production. Symptoms may include reduced muscle mass, greater height, poor coordination, less body hair, smaller genitals and gynaecomastia.

\section{Case report}

An 18-year old young man was followed due to Klinefelter and received androgen replacement since the age of 12 . As he was shifted to IM injections his liver enzymes increased and liver damage due to androgen replacement was suspected. A liver biopsy showed steatosis and, accordingly, androgen replacement was paused.

However, the medical history revealed $20 \mathrm{~kg}$ weight gain from the age of 16 to 18, abdominal striae and mental changes, leading to evaluations for CS. Initial tests showed elevated urinary free cortisol levels of 213, 238 and $397 \mathrm{nmol} /$ day (ref: 12-150) and borderline abnormal $1 \mathrm{mg}$ dexamethasone suppression test (DST).

Over the following months, equivocal test results challenged the clinicians. Repeated long DSTs were normal, urinary free cortisol levels were fluctuating and, subsequently, a peripheral $\mathrm{CRH}$ test showed more than $50 \%$ rise in ACTH. Finally, MRI demonstrated a 5 $\mathrm{mm}$ hypointense mass in the pituitary gland and an inferior petrosal sinus sampling showed a pituitary-to-peripheral ACTH-response $>5$, which led to the diagnosis of Cushing's disease.

Two years after first CS suspicion a transsphenoidal adenomectomy was performed. Subjectively, he felt better after the operation. Unfortunately, histology did not reveal signs of an adenoma and during the following years he presented with clinical CS features again. He resumed androgen replacement to rule out androgen deficiency as responsible for the symptoms, and liver function tests were unrelated to this. Repeated DSTs showed normal suppression of cortisol despite several elevated urinary free cortisol levels.

Thus, the cyclic manifestation of Cushing's had, very likely, relapsed. The patient had side effects to all possible registered medical therapies for Cushing's and refuses another neurosurgical procedure.

\section{Conclusions}

This case illustrates how overlapping symptoms of cyclic CS and hypogonadism due to lack of androgen replacement further hamper diagnosis and management of cyclic CS.

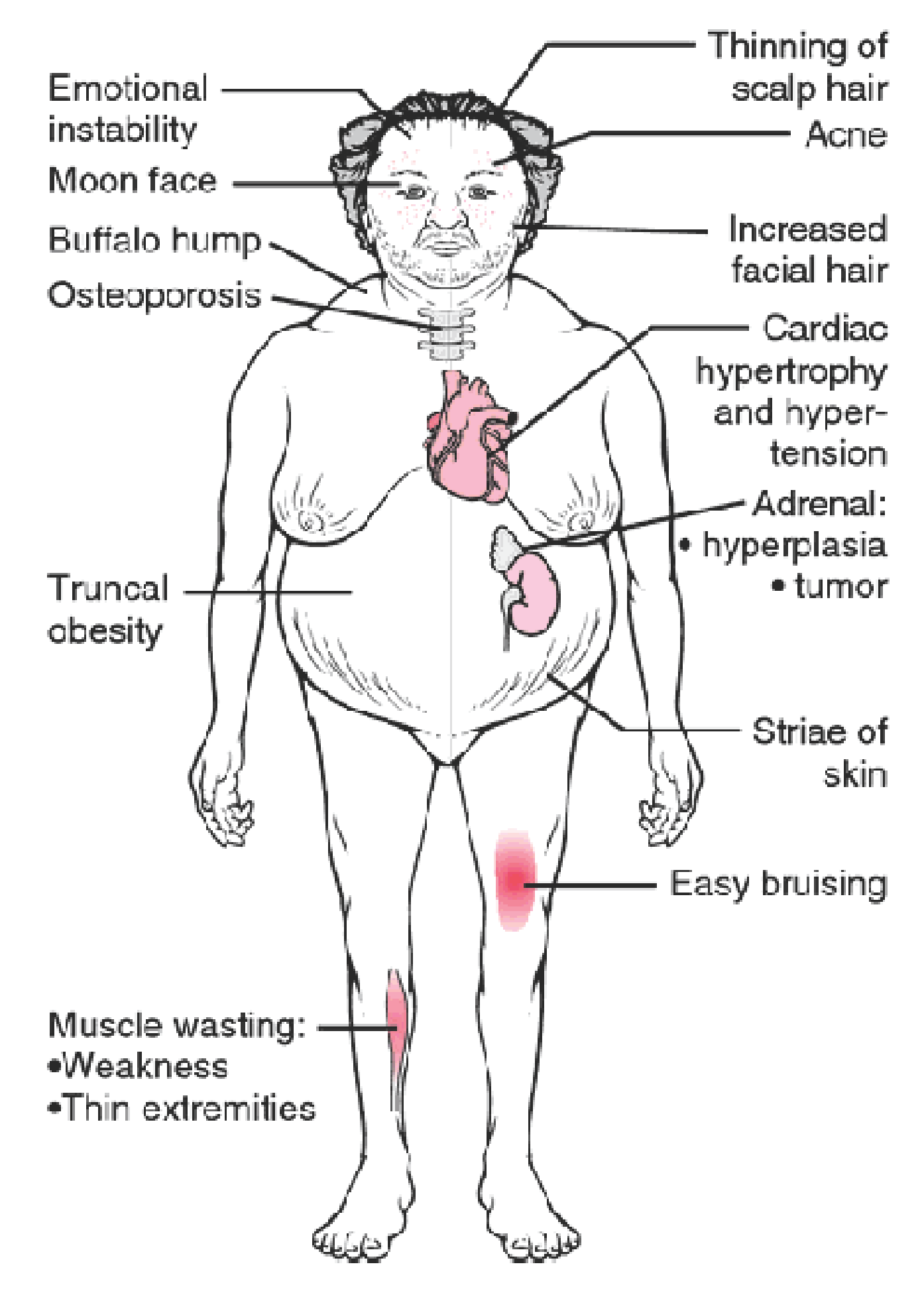

Characteristic features of Cushing's

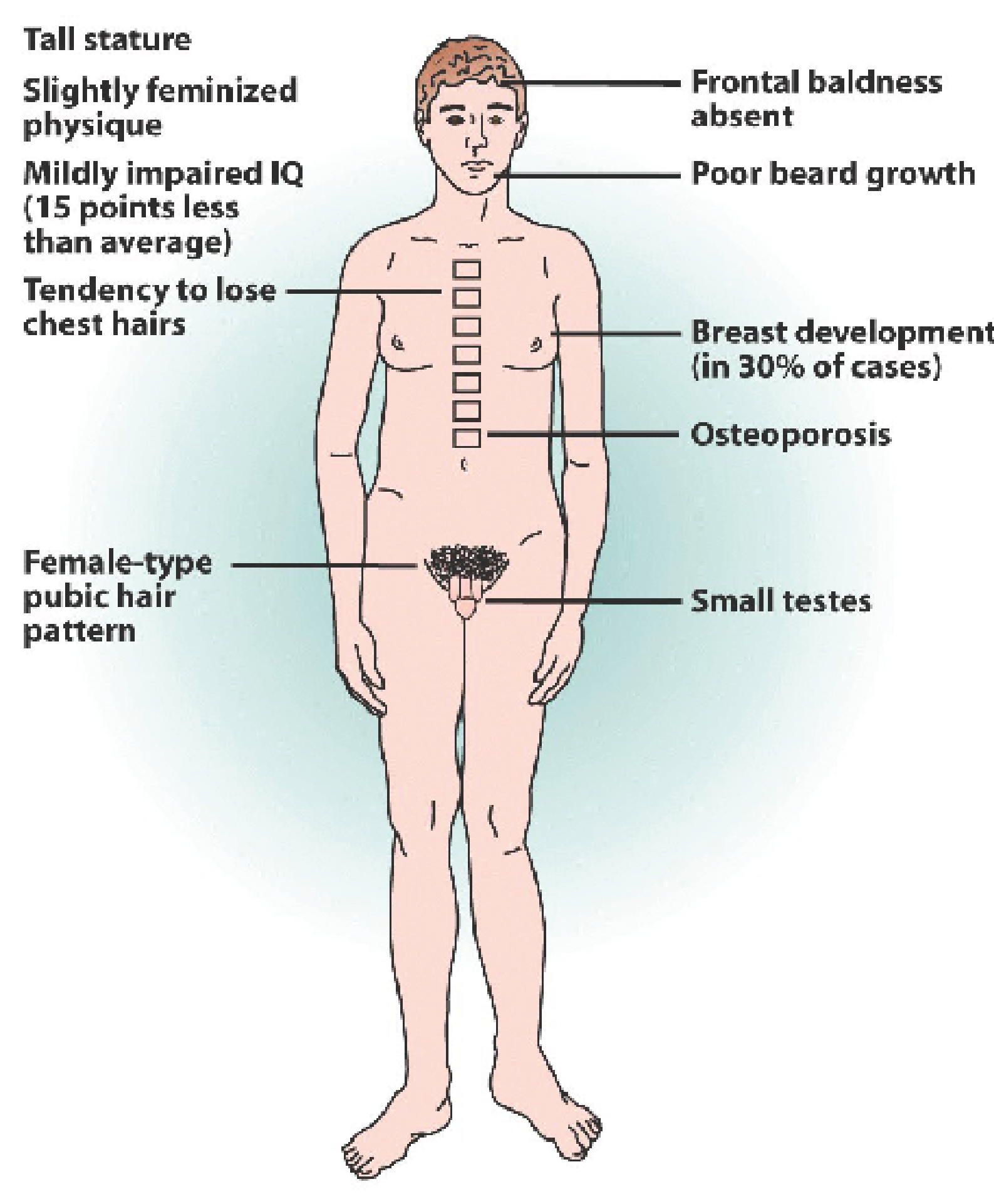

Characteristic features of Klinefelter
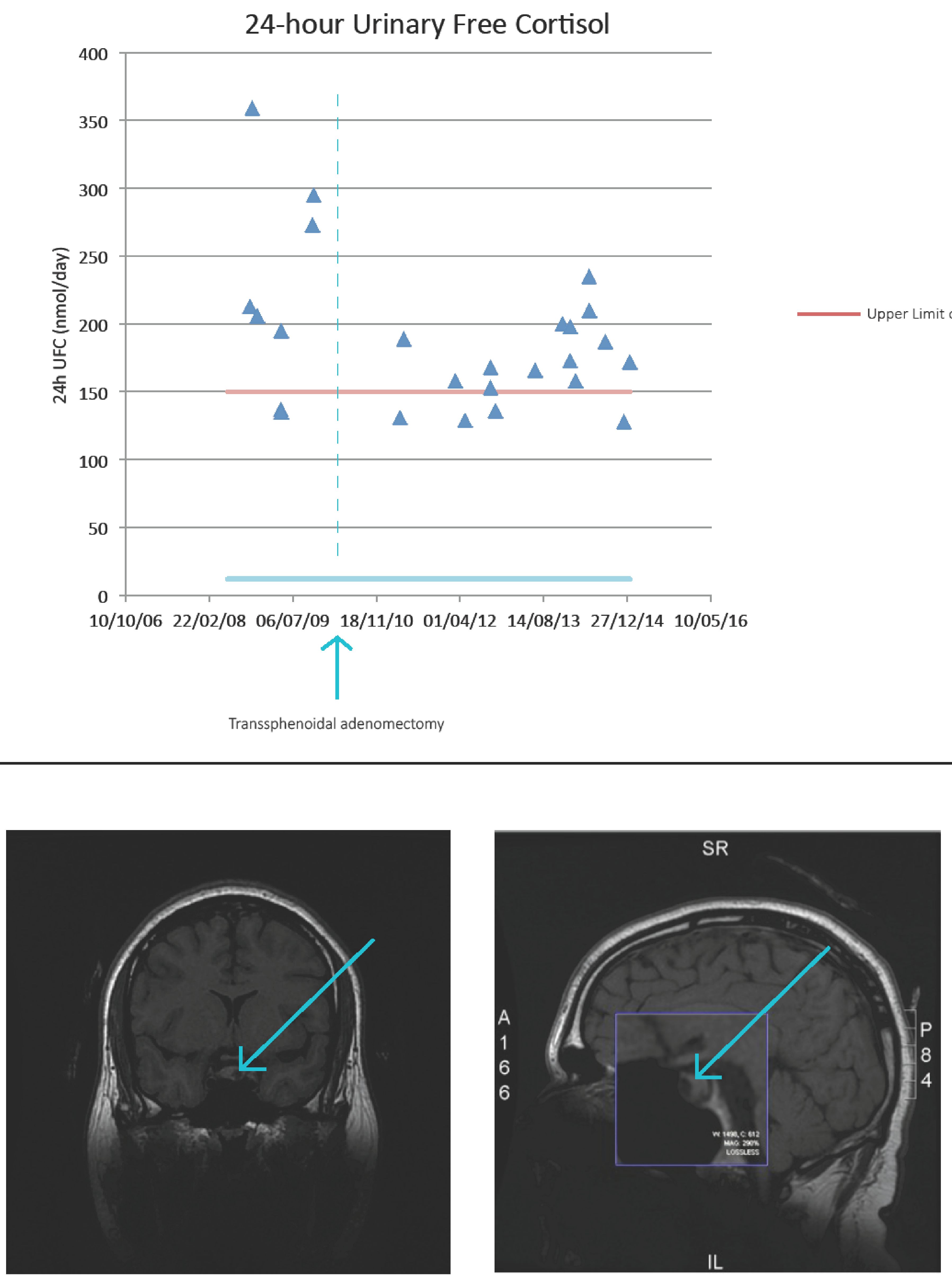

MRI showed a $5 \mathrm{~mm}$ hypodense mass in the pituitary gland. 